\title{
Relationship between attenuation of coda wave and crustal stress
}

\author{
Kyosuke OKAMOTO ${ }^{1}$, Hitoshi MIKADA² Tada-nori GOTO² and Junichi TAKEKAWA ${ }^{2}$ \\ ${ }^{1}$ Dept. of Civil and Earth Res. Eng., Kyoto University, JSPS Research Fellow \\ ${ }^{2}$ Dept. of Civil and Earth Res. Eng., Kyoto University.
}

\begin{abstract}
Stress field deep under the ground, like seismogenic depth, is important to know seismic activity, state of oil reservoir, etc. However we can only know stress field near the earth surface using the current equipment and technique. In this study we focus on seismic scattering waves having information of the earth crust where the seismic waves travel through. From the scattering waves, we try to estimate the stress field deep under the ground. At first we employ a 2-D finite different method to reveal a relationship between the seismic scattering and the crustal stress field, i.e., magnitude and direction of the stress. As the result, it is revealed that coda- $Q$, which is derived from attenuation ratio of a seismic wave, has a proportional relationship with magnitude of the stress and changes periodically against direction of the magnitude. Next it is examined if the relationship can be seen in real field data using seismograms obtained by Hi-net. And it is revealed that coda- $Q$ has the relationship with change in strain obtained by GPS observation and theoretical dislocation calculated from fault movement; both are proxy of the crustal stress field. We conclude that change in crustal stress field can be estimated from monitoring of coda- $Q$.
\end{abstract}

\section{INTRODUCTION}

In research of earthquake occurrence, it is important to monitor the change of crustal stress field in the seismogenic zones. However, it is difficult to measure the stress at seismogenic depth because of limitation of equipment ability, cost of drilling, and so on.

Recently, Hiramatsu et al. (2010) stressed that coda- $Q$ (denoted as $Q_{c}$, hereafter) is a parameter of the differential strain rate, which relates with the stressing rate of shear stress, from the observation of the $Q_{c}$ in the vicinity of the 1995 Southern Hyogo Prefecture earthquake before and after the main shock event. Sugaya et al. (2009) discussed a change in $Q_{c}$ after the 1995 Southern Hyogo Prefecture earthquake in connection with a property change in the crust, particularly in the lower crust. The cracks opened or closed by the stress change are one of the major factors which change the property of the crust. Jin et al. (2004) and Aki (2004) proposed a "Brittle-Ductile Hypothesis" after a long-term observation of the seismicity around the San Andreas Fault that has led them to find a high correlation with $Q_{c}$.

The parameter $Q_{c}$ is derived from attenuation ratio of energy in a coda wave, which origin is residual vibration caused by inhomogeneities around the seismic source. Some papers reported anomalous value of $Q_{c}$ around volcanoes, faults
(Matsumoto and Hasegawa, 1989), which means the media has anomalous inhomogeneity. Also the recent results (Hiramatsu et al., 2010) reported that anomalous value of $Q_{c}$ was detected after a large earthquake. These results suggest that the order of inhomogeneities may vary in the course of long-term earthquake generation cycle, i.e., before and after the failure of crustal material takes place. Here we make a reasonable deduction with fair certainly that the stochastic parameter $Q_{c}$ can have a certain relationship with physical or state properties of the stress field in the particular part of the crust where seismic waves propagate through, since the change in the stress filed is a trigger of earthquakes.

In this paper, we employ numerical simulation schemes to test our hypothesis that $Q_{c}$ would vary with change in the stress acting on the medium where seismic waves travel through. We reveal a relationship between $Q_{c}$ and crustal stress. Also we consider if there is a relationship between $Q_{c}$ calculated from field data and the strain obtained by GPS measurement, which is considered as a proxy of the crustal stress at near surface.

\section{NUMERICAL SIMULATION}

We employ a 2-D Finite Different Method (FDM) with discontinuous grid to simulate propagating and scattering elastic waves in the crust. Equation of motion and stress-strain relation for 
2-D SH wave are discretized by a rotated staggered grid (Saenger et al., 2000).

Fig. 1 shows a simulation model. The upper crust is discretized by the FDM mesh which interval is three times as fine as that of the lower crust. Using this mesh, detailed shape of the cracks in the upper crust, where larger cracks distribute than in the lower crust, can be described. We assume that plane wave incidents from the bottom of the lower crust. A wave form of the plane wave is the Ricker wavelet with the central frequency of $9 \mathrm{~Hz}$. We assume that the initial model is stable under the initial confining pressure. In addition to the initial confining pressure, normal stress whose magnitude ranges from $-4 \mathrm{MPa}$ to $16 \mathrm{MPa}$ is loaded along the $x$ or $z$ axis. The cracks close and open selectively by the additional stress, and then deflector arrangement of the cracks and anisotropy on elastic wave velocity filed occur (Nur, 1971) in the upper crust. The cracks satisfying Eq. 1 close when the compression stress is loaded and the cracks satisfying Eq. 2 newly open when the tensile

$$
\begin{aligned}
\text { stress is loaded. } & \alpha \leq \sigma_{n} / E \\
& \alpha>\sigma_{n} / E
\end{aligned}
$$

where $\alpha$ is crack aspect ratio, $\sigma_{n}$ is magnitude of stress normal to a crack and $E$ is the Young's module. The number of newly opening cracks due to the tension is same as the number of closed cracks when the same magnitude of compressive stress is loaded. These equations are obtained by a rock experiment. However, here we assume that they can be applied to larger scale, the crust. We also consider new opening cracks at the edges of the closed cracks due to incensement of deviatoric stress (Sato, 1992). Length of the new opening cracks is far shorter than that of the original crack.

A seismogram is recorded at the stations located

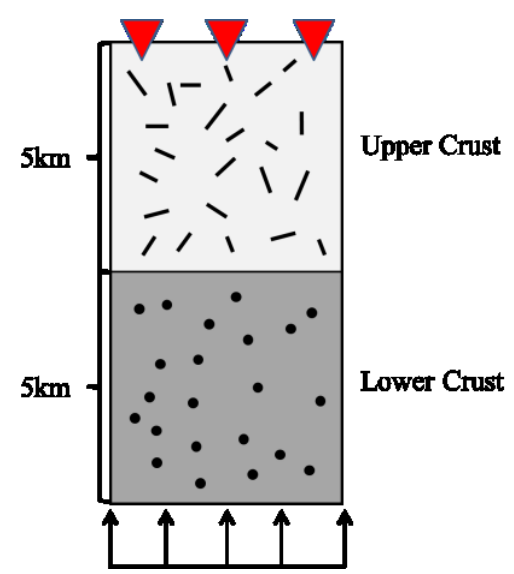

Fig. 1 Plane wave incidents from the bottom of model. In the upper crust, 600 line shaped cracks distribute, and in the lower crust 1,000 point shaped cracks distribute. on the surface of the model before and after the stress is loaded. From this seismogram, $Q_{c}^{-1}$ (the reciprocal of $Q_{c}$ ) is calculated after fitting Eq. 3 to envelope of the coda wave $e(t)$.

$$
e(t)=A_{0} t^{-n} \exp \left(-\frac{\omega}{2 Q_{c}} t\right)
$$

where $A_{0}$ is initial amplitude, $t$ represents the time from origin of an earthquake and $\omega$ is angular frequency. The term of $t^{-n}$ represents geometrical spreading. Power $n$ is from 1 to 2 depending on the dominance wave, i.e., surface, diffusive or body waves, in practice. However in this study we set $n=$ 0 because a plane wave, which does not spread, is assumed as the incident wave. Higher $Q_{c}^{-1}$ is observed when the coda wave strongly attenuates.

\section{VARIATION IN $Q_{c}^{-1}$ AGAINST CRUSTAL STRESS}

Fig.2 shows variation in $Q_{c}^{-1}$ against magnitude of the loaded stress. The $Q_{c}^{-1}$ increases in both cases, when the stress is loaded along the $x$ or $z$ axis. However magnitude range where the $Q_{c}{ }^{-1}$ increases is different between the case of the $x$ and $z$ axes loading. This increase is due to change in (1) reflection pattern at the cracks, (2) elastic wave velocity field and (3) number density of the cracks. Little energy in the incident wave transfers to the coda wave when direction of a crack coincides with the incident wave (the case of the stress is loaded along the $z$ axis). It makes the coda wave attenuate and high $Q_{c}^{-1}$ is observed. Also high $Q_{c}^{-1}$ is observed when elastic velocity is faster because the coda wave attenuates much rapidly against time. When number density of the cracks decreases, high $Q_{c}^{-1}$ is also obtained because little energy transfers to the coda wave by scattering from the incident wave. In brief conclusion, $Q_{c}^{-1}$ increases when the stress is loaded regardless its direction but the magnitude range within that the $Q_{c}^{-1}$ increases has dependence on direction of the loading. $Q_{c}^{-1}$ increases when the confining pressure increases.

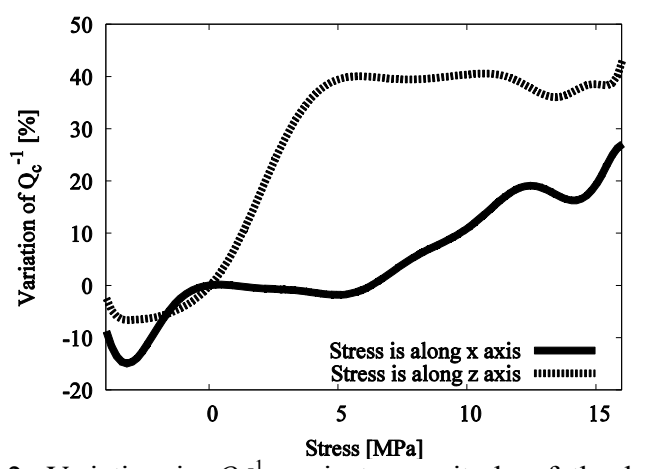

Fig.2: Variation in $Q_{c}^{-1}$ against magnitude of the loaded stress. The stress is loaded along $x$ or $z$ axis. $Q_{c}^{-1}$ at $0 \mathrm{MPa}$ is a standard value for the variation ratio. 

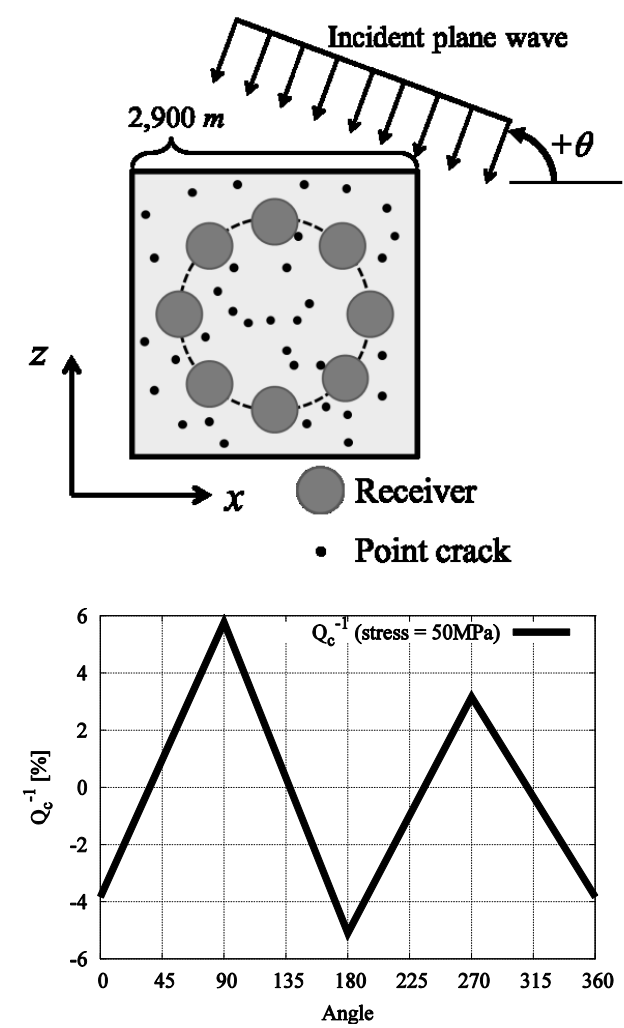

Fig. 3 (Upper) The model which is used to examine arelationship between $Q_{c}^{-1}$ and direction of stress. Direction of the incident wave changes, with direction of the stress, location of the receivers fixed. $Q_{c}^{-1}$ is taken average over the model using the receivers. (Lower) Variation in $Q_{c}^{-1}$ against direction of the incident wave.

We also examine a relationship between $Q_{c}^{-1}$ and stress direction. For this objective, we employ a model shown in Fig.3 (Upper). We fix direction of the loaded stress (principal stress is along $z$ direction) and location of the receivers and then change direction of the incident wave. As a result, it is found that $Q_{c}{ }^{-1}$ changes periodically against the direction of the incident wave. The $Q_{c}^{-1}$ marks the maximum value when direction of the incident wave corresponds with that of the loaded stress.

\section{FIEDLD DATA ANALYSYS}

The followings are revealed from the numerical simulations,

1. $Q_{c}^{-1}$ has a proportional relationship with the magnitude of stress.

2. $Q_{c}^{-1}$ marks the maximum value when the direction of the incident wave corresponds with that of loaded stress.

Using $Q_{c}^{-1}$ calculated from earthquakes recorded by Hi-net (Okada et al., 2004) and strain obtained by GPS observation (Miyazaki et al., 1998), we examine if the relationship can be seen in field data. Detailed information of the observed earthquakes is below;

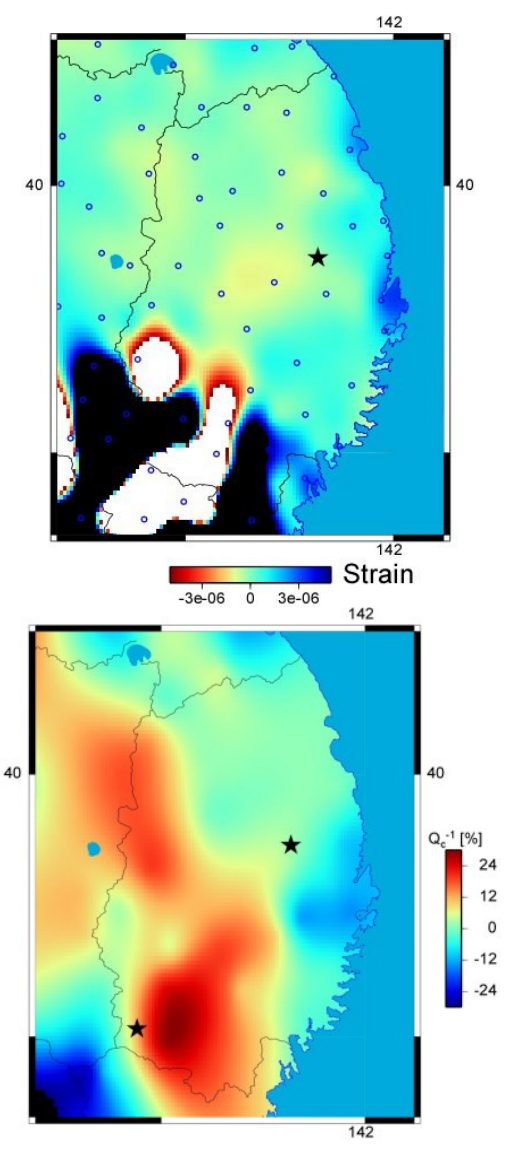

Fig. 4 (Upper) Variation in strain obtained by GPS before and after the Iwate-Miyagi Nairiku earthquake in 2008 and the Northern Iwate earthquake in 2008 (both are shown by $\star)\left(\right.$ White $=$ strain less than $-5.0 \times 10^{-6} 、$ Black $=$ strain more than $\left.5.0 \times 10^{-6}\right)$. (Lower) Variation in $Q_{c}^{-1}$ at the same period.

- Analyzed area: Iwate, Tohoku

- Observation term: Jun. 2006 - Jun. 2010

- Distance between hypocenter and station: within $40 \mathrm{~km}$

- Magnitude: $1.5-7.0$

- Depth: shallower than $100 \mathrm{~km}$

- Target frequency: $6 \mathrm{~Hz}$

Within the observation term, two major earthquakes occurred. One is the Iwate-Miyagi Nairiku Earthquake in 2008 (Jun. 14, 2008, $M_{w}$ 7.2, depth $7.8 \mathrm{~km})$ and the other is the Northern Iwate Earthquake in 2008 (Jul. 24, $M_{w} 6.8$, depth $108 \mathrm{~km}$ ). We took average of $Q_{c}^{-1}$ at each station respectively before and after the earthquakes; May, 2006 - May, 2008 and Oct, 2008 - Jan 2010. Then, we calculate the difference between these two sets of the averaged $Q_{c}^{-1}$ (Fig. 4). The variation in $Q_{c}^{-1}$ shows the tendency obtained by the numerical simulation $\left(Q_{c}^{-1}\right.$ increases when the region is shortened and decreases when tensed) around the two hypocenters. There are regions where the tendency is not seen. This is because the $Q^{-1}$ and the strain obtained by GPS is affected by different depth each other.

Next, we confirm the other finding revealed by the numerical simulation; $Q^{-1}$ marks the maximum 


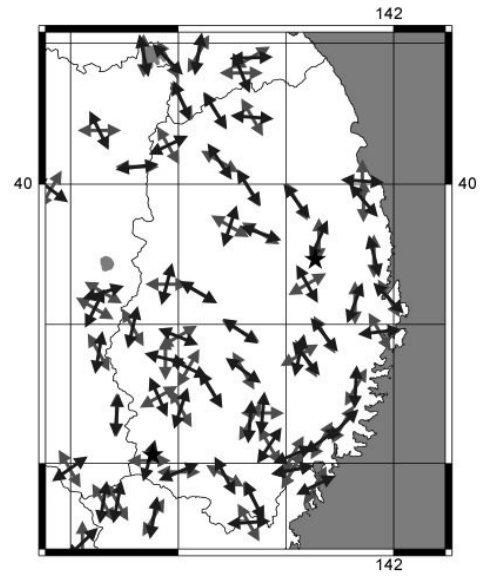

Fig. 5 Direction of the maximum strain obtained by GPS (the gray arrows) and direction of the maximum $Q_{c}^{-1}$ (the black arrows). Set of $\star$ are hypocenter of a couple of earthquake mentioned in Fig.5.

value when direction of incident wave corresponds with that of principal stress. Fig.5 shows direction of the maximum strain obtained by GPS observation and maximum $Q^{-1}$. Directional dependence of $Q^{-1}$ is calculated by choosing the analyzed earthquakes in terms of angle from a station. The direction of maximum strain coincides with that of $Q^{-1}$ along the southern coast of Iwate. It means that the $Q^{-1}$ indicates the direction of maximum stress at the surface. However at some points, these two directions $\left(Q^{-1}\right.$ and strain) are different. It is because the $Q^{-1}$ indicates state of subsurface between hypocenter and station while strain obtained by GPS observation indicates sate only at the surface.

\section{SUMMARY}

We hypothesized that $Q$ value has a relationship with stress, which is a trigger of earthquake, and examined using the numerical simulation. As the result, it is revealed that $Q^{-1}$ has a proportional relationship with magnitude of stress and changes periodically against direction of stress. Then we confirmed that this relationship can be seen in real field data.

As a future work, we confirm if the relationship can be seen at other regions, e.g., around the Surugawan earthquake in 2009, the Chuetsu earthquake in 2004, etc.

ACKNOWLEDGMENT: We thank Japan Society for the Promotion of Science (JSPS) for the Grant-in-Aid for JSPS Fellows $(24 \cdot 373)$ for supporting the present research. We also thank National Research Institute for Earth Science and Disaster Prevention (NIED) and Geospatial Information Authority of Japan (GSI) to make their data available and Prof. Ru-shan Wu (University of
California, Santa Cruz) for important comment and suggestion.

\section{REFERENCES}

1) Aki, K., 2004, A new view of earthquake and volcano precursors, Earth Planets Space, 56, 689-713.

2) Hiramatsu, Y., K., Iwatsuki, S., Ueyama, and T., Iida, 2010, Spatial variation in shear wave splitting of the upper crust in the zone of inland high strain rate, central Japan, Earth Planets Space, 62, 675-684.

3) Jin, A., K., Aki, Z., Liu, and V. I.,Keilis-Borok, 2004, Seismological evidence for the brittle-ductile interaction hypothesis on earthquake loading, Earth Planets Space, 56, 823-830.

4) Matsumoto, S., and Hasegawa, A., 1989, Two-dimensional coda Q structure beneath Tohoku, NE Japan, Geophys. J. Int., 99, 101-108.

5) Miyazaki, S., Y., Hatanaka, T., Sagiya, and T., Tada, 1998, The Nationwide GPS array as an Earth observation system, Bull. Geogr. Surv. Inst., 44, 11-22.

6) Nur, A., 1971, Effect of Stress on Velocity Anisotropy in Rocks with Cracks, J. Geophys. Res., 76, 2022-2034.

7) Okada, Y., Kasahara, K., Hori, S., Obara, K., Sekiguchi, S., Fujiwara, H., and Yamamoto, A., 2004, Recent progress of seismic observation networks in Japan-Hi-net, F-net, K-NET and KiK-net-, Earth Planets Space, 56, 15-28.

8) Saenger, E. H., N., Gold, and S. A., Shapiro, 2000, Modeling the propagation of elastic waves using a modified finite-difference grid, Wave Motion, 31, 77-92.

9) Sato, Y., 1992, Theoretical study on dilatancy of dry crack, J. Soc. Mat. Sci., Japan, 41, 1068-1074 (in Japanese with English abstract).

10) Sugaya, K., Y., Hiramatsu, M., Furumoto, and H., Katao, 2009, Coseismic change and recovery of scattering environment in the crust after the 1995 Hyogo-ken Nanbu Earthquake, Japan, Bull. Seism. Soc. Am., 99, 435-440. 